\title{
Venous thromboembolic disease
}

\section{in the older adult patient}

Enfermedad tromboembólica venosa en el paciente adulto mayor

Doença tromboembólica venosa em paciente idoso

José Javier Elizalde González*

There is no doubt about the relevance and importance of Venous thromboembolic disease (VTE) in current medicine, both because of its frequency (more than 750,000 new cases per year in the European Community), and because of its high recurrence when anticoagulation is stopped (11\% per year, $20 \%$ at two years, $29 \%$ at five years and $40 \%$ at ten years), as well as its high mortality (greater than the sum of the mortality from motor vehicle accidents plus the mortality of breast cancer, plus HIV-AIDS mortality, plus prostate cancer mortality, yes all together!), as well as its long-term sequelae in the form of venous insufficiency and post-thrombotic syndrome and chronic thromboembolic pulmonary hypertension, as well as its escalating costs of care (of up to US \$2 million in annual hospital expenses per institution). It has been the third most common cardiovascular disease for years after acute coronary syndromes and cerebrovascular disease, enough arguments to be of interest to any doctor, particularly the specialist in critical care medicine, every time the elderly are $48 \%$ of the critically ill population in ICUs and will continue to represent a substantial proportion of patients in the ICU as the general population ages and the evidence increases in the sense that age is not an independent predictor of outcome in ICU, in fact in the series of multivariate logistic regression equations of the APACHE III prognostic system for ICU patients, after adjusting for different variables, age only explains $3 \%$ of the variance of the interpatient differences in the risk of death, being the major explanatory variable the acute physiology score.

Despite the high prevalence of VTE in older patients, relatively little is known about the clinical aspects of the disease in this specific subset of the population, an important aspect given the gradual aging of humanity. It has been observed that the population over 85 years of age in the USA went from 3 million in 1990 to 7 million this year, a figure that will double by 2040 , in such a way that by 2050 , it is estimated that the $5 \%$ of the total population will exceed this age.

\footnotetext{
${ }^{*}$ Editor, INCMNSZ.
}

Citar como: Elizalde GJJ. Venous thromboembolic disease in the older adult patient. Med Crit. 2020;34(5):262-264. https://dx.doi.org/10.35366/96455

www.medigraphic.com/medicinacritica
At this time a quarter to a fifth of the population of Europe is older than this age, one of the explanations for the high mortality in the first wave of COVID-19 in some countries in the area.

Nations historically considered as «young» due to the large population base of children and young people like Mexico are also facing a progressive process of population aging. The country's population over 65 years of age went from 7 million in 2010 to almost 7,750,000 inhabitants in 2013 and a year later in 2014 it already had more than 8 million inhabitants, an increase of $22 \%$ in this segment of the population in just 3 years. We do not have data from this year's census in the country due to the complications derived from the SARS-CoV-2 pandemic, but according to data from the 2018 National Survey of Demographic Dynamics, carried out by the INEGI, the number of Mexicans of more than 60 years amounts to 15.5 million, representing more than $12 \%$ of the total population. The percentage of mortality due to diseases in this group over 65 years of age is $75 \%$ for the circulatory system and $72 \%$ for the respiratory system, and 54 out of 100 deaths in Mexico occur in people in this age group.

The known case fatality rate from pulmonary embolism (PE) increases exponentially with age from $3.6 \%$ in patients aged 25 to 34 years to $17.4 \%$ in patients older than 85 years.

We can find the association between VTE and advanced age at many levels, for example as a risk factor for the first episode of this disease, and as a risk factor for the development of complications after a thrombotic event, there is a lot of literature in this regard; the incidence of VTE increases steeply with age. The diagnosis of $\mathrm{PE}$ in those over 70 years is 6.2 times higher than in young people and the deep venous thrombosis (DVT) is 12.7 times more frequent between 70 and 79 years vs 20 to 29; generating a slight exponential curve, without a cut-off point where there is no risk. In this regard, there are probably underlying racial and genetic factors as this association is less intense in the Asian population.

Using a multivariate analysis of proportional hazards regression, we found in the ICOPER Registry a hazard ratio of $1.6(95 \% \mathrm{Cl}, 1.1-2.3)$ for the age of over 70 years. When analyzing the age distribution in the same registry, we found a mean age of $62.3 \pm$ SD 16.7 years, with a median of 66 years and a range that spanned from 4 months 
to 99 years; $24 \%$ of the patients corresponded to the age group between 60 and 69 years, $25 \%$ between 70 and 79 years and $14 \%$ were older than 80 years of age.

If we focus on the pathophysiological mechanisms of a high-risk PE, it is easy to find that at a certain age the impact of factors that eventually lead to death through cardiogenic shock, such as increased wall tension, ischemia of the right ventricle with a drop in cardiac output and secondary hypoperfusion to mention just a few will be greater, and the various compensatory mechanisms will be evoked late or partially at best.

One of the most widely used scoring systems globally, the original PESI (Pulmonary Embolism Score Index) includes among its variables with the greatest weight precisely the age expressed in years and exactly above 60 , age is the most important one.

Although without completely solid evidence within the natural history of the disease, age, male sex, family history of VTE, and increased body mass index show an independent association with late disease recurrence.

The reason to explain this higher incidence and prevalence of VTE in the elderly is not entirely clear, but it is surely multifactorial, the hypercoagulant state related to age, sedentary lifestyle, greater comorbidity, and endothelial injury, decreased blood flow, low cardiac output, abnormal activation of various coagulation factors and the well-known geriatric frailty syndrome, among others.

Thus, the implications of PE in the elderly include epidemiological aspects (incidence, prevalence, morbidity, mortality), as well as clinical (comorbidity that influences signs and symptoms, differential diagnosis), and diagnostic (specificity of D-Dimer, EKG, echocardiogram, chest X-ray and lung scans). It seems that single detector and multidetector pulmonary angio-CT are not directly influenced by age, but its use is limited in the elderly because of the higher risk of side effects, especially nephrotoxicity compared with younger patients.

One of the most notable findings of the RIETE registry in a group of 858 nonagenarians is that VTE is a problem of women (only 20 to $28 \%$ of this population were men), as expected its underlying conditions: chronic heart disease, chronic lung disease, cancer and abnormal levels of creatinine. Their risk factors: recent immobility in about $40 \%$ (of 4 or more days), followed by a history of VTE and recent surgery. They had their treatment based on LMWH in $90 \%$ of the cases, followed by vitamin $\mathrm{K}$ antagonists and the same $\mathrm{LMWH}$ in similar proportions, with a mortality of $21 \%$ for DVT and $33 \%$ for $\mathrm{PE}$, being the most frequent cause of death $\mathrm{PE}$ itself by up to $11 \%$, followed by respiratory failure and infection. Observing the characteristics of a subgroup of 351 nonagenarians with acute VTE secondary to immobility greater than 4 days, it was found that the main reason for this immobility was dementia and depression (30-43\%), followed by infection (14-17\%) and less frequently heart failure and trauma. The duration of immobility was very long, from 1 to 4 weeks in just over $30 \%$ of the cases and more than 8 weeks in up to $44 \%$, having occurred primarily at the household level.

However, the probably most significant contribution is the finding of the very low frequency of pharmacological thromboprophylaxis in the elderly, since it was found that only around $20 \%$ of the elderly received this benefit, much work needs to be done in this regard to provide better prevention to this segment of the population that represents the largest group of patients with VTE.

Some systematic reviews of the literature reach the same conclusions, one condition more of elderly women, with hospital-acquired events, finding dyspnea as the most common symptom and risk factors such as bed rest, previous DVT, cancer, chemotherapy, and surgery (provoked events in up a 52\%), EKG and plain chest film normal up to $50 \%$ and $62 \%$ of cases respectively, with a mean $\mathrm{PaO}_{2}$ in the range of 53 to $61 \mathrm{mmHg}$ and a mean $\mathrm{Da}-\mathrm{aO}_{2}$ in the range of 45 to $47 \mathrm{mmHg}$.

In the Worcester VTE Study, the reported incidence rates of VTE were increased more than 10-fold in individuals aged 75 years or more, compared with patients of less than 55 years. Older patients represented more than $60 \%$ of all cases of VTE occurring in residents of this American county in Massachusetts.

Advancing age is also associated with an increased risk of all-cause mortality. Mortality rates are $9.9 \%$, $28.4 \%$, and $37.0 \%$ in elderly subjects at 30 days, 1 -year, and 3-year follow-up. In multivariable Cox regression models, age superior to 80 years has been associated with all-cause mortality (HR 1.74; 95\% confidence interval $(\mathrm{Cl}), 1.43-2.11)$.

We know that with advanced age, patients are more likely to develop the so-called provoked VTE. It has been observed that many elderly patients with provoked or unprovoked forms of VTE have been anticoagulated for more than 3 months or more than 12 months, respectively. Interesting and against logic advanced age is not especially associated with an increased risk of recurrent VTE (3.0, 7.8, and 9.5\%, respectively, at day 30, 1-year, and 3-year follow-up, and those cases specifically characterized as PE occurred in just 1.1, 3.0, and $3.6 \%$, respectively), but elderly patients, in general, have a higher risk of bleeding from continued anticoagulant therapy (major bleeding rates of $5.2 \%, 9.0 \%$, and $10.6 \%$ has been observed in elderly subjects at day 30 , 1-year, and 3-year follow-up, respectively) such clinical practice is potentially harmful to this segment of the population. Using Cox regression modeling, the age of more than 80 years is associated with a trend toward an increased risk of bleeding (HR 1.42; 95\% Cl, 0.98-2.07), compared with patients $<80$ years.

The catastrophic scenario of an emergency due to a state of shock secondary to a high-risk PE in an elderly 
patient exists and in light of this, the analysis on the effectiveness and safety of thrombolysis in this subgroup is appropriate. We know that bleeding phenomena after systemic infusion of lytic therapy are more frequent in older individuals, increasing age is a major risk factor for hemorrhagic complications after pulmonary embolism thrombolysis. It has been found when separating age groups that the crude RR, $95 \% \mathrm{Cl}$ of bleeding due to thrombolysis for individuals under 50 years of age is 1.0 , while when considering those between 50 to 70 years this practically doubles, rising to $1.9(0.9-4.0)$, but in the case of people over 70, it almost triples to 2.7 (1.3-5.6), $(1.0,2.0(0.9-4.2)$ and $3.9(1.7-8.9)$ respectively in a multivariate-adjusted $\mathrm{RR}, 95 \% \mathrm{Cl}$ ).

A final consideration of the use of the new directacting oral anticoagulants generally known as NOACs in elderly patients. As we know polypharmacy, co-morbidities, and altered pharmacokinetics, often present in elderly patients render the use of anticoagulants quite challenging, being the NOACs good alternatives to the common vitamin $\mathrm{K}$ antagonists, taking advantage of their fewer drug and food interactions, general ease of use and superior compliance.

There are some major studies with these new molecules, dabigatran, rivaroxaban, apixaban, and edoxaban that included numbers of patients above 75 years of age. Taking into account the potential problems in advanced age, such as a higher frequency of kidney failure, a lower body mass index due to a different muscle/ fat distribution, greater comorbidity, polypharmacy, and reports of bleeding, there is evidence that the benefit of NOACs is consistent among all age groups, featuring equal or greater efficacy in preventing thrombotic events and with less bleeding complications (especially with edoxaban and apixaban), although a head to head comparison doesn't exist. Therefore, we need to proceed with caution, always carrying out a risk-benefit analysis on a case-by-case basis.

It is clear that this is a relevant issue in light of the aging of world society and that it still leaves many questions to be answered; the medical team will often have to make difficult decisions at the bedside of the elderly patient with VTE and other medical problems for their best benefit. The message is to carry out more and better research on all these aspects of the elderly and build better scientific evidence preferably before being on the other side of the barrier. The contributions from the critical care community will be always welcome.

Correspondence:

José J Elizalde González, M.D.

E-mail: jjeg@unam.mx 